\title{
Capacity Limitations of Medical Services Provided by Pediatricians in Japan
}

\author{
Jun Kohyama \\ Tokyo Bay Urayasu Ichikawa Medical Center, Urayasu, Japan \\ Email: j-kohyama@jadecom.or.jp
}

Received 15 February 2014; revised 10 March 2014; accepted 17 March 2014

Copyright (C) 2014 by author and Scientific Research Publishing Inc.

This work is licensed under the Creative Commons Attribution International License (CC BY). http://creativecommons.org/licenses/by/4.0/

(c) (i) Open Access

\begin{abstract}
The shortage of pediatricians has been identified as one of the largest social problems in Japan. This study aimed to compare the assumed ideal nighttime working conditions of pediatric hospitalists in Japan with the actual current situation. By using available data on the age-related numbers of pediatricians in Japan, we described the ideal working conditions for pediatric hospitalists in Japan. The ideal situation was then compared with the existing actual working conditions. Compared with the assumed ideal condition, current pediatric hospitalists are working in many hospitals with a few colleagues. To solve the current supply-demand mismatch on medical care for children during night, an involvement of non-pediatric physicians in the medical care of children during the night is indispensable. Also, parents in Japan need to know the limitations relating to the available capacity of pediatricians, and accept that their children can be cared for adequately by non-pediatric physicians, who have been trained to provide primary medical care to children.
\end{abstract}

\section{Keywords}

Pediatric Hospitalist; Night-Duty; Social Needs; Non-Pediatric Physician; Parents

\section{Introduction}

The shortage of pediatricians has been identified as one of the largest social problems in Japan [1] [2], but an effective solution has not been provided. There are ongoing frustrations from parents and pediatricians about this issue; pediatricians often claim that they are busy, and parents wish for their children to be cared for by pediatricians. In Japan, the medical insurance system guarantees the free access to healthcare. In addition, local government officials, especially mayors, provide a certain degree of free care for children as a way to ensure support from their constituents. Both of these situations have increased demand for care and highlighted issues relating to the lack of pediatricians. To secure adequate working conditions for pediatricians, the Japan Pediatric 
Society (JPS) has recommended that pediatricians who have engaged in overnight work, should be absent from work after overnight duties, and that hospitals who accept child patient admissions should employ at least 10 pediatricians [1]. JPS also states that children should be cared for by pediatricians [1].

From a different point of view, it should be noted that sleep loss impairs brain function [3]. In middle-aged subjects aged 37 to 52 years, a phase shift produces a greater reduction in sleep duration and efficiency than in younger people (aged 18 to 25 years) [4]. The night work burden should be reduced for older people in order to obtain sufficient performance from them. This paper aimed to assess the ideal working conditions for Japanese pediatric hospitalists with paying special interest in their night work burden.

\section{Materials and Methods}

By using currently available recent data on the age-related numbers of pediatricians in Japan [5], and taking into consideration acceptable overnight working conditions as well as the recommendations of JPS, we described the ideal working conditions for pediatric hospitalists in Japan. This ideal situation was then compared with the existing actual working conditions.

\section{Results}

In 2008, the Ministry of Health, Labour and Welfare (MHLW) surveyed 9308 pediatric hospitalists [5]. To summarize the available medical services during night provided by pediatric hospitalists, several assumptions were made.

The age of pediatricians involved in overnight work ranged from 24 to 59 years, the frequency of overnight work was highest in workers in their $20 \mathrm{~s}$ and $30 \mathrm{~s}$, and then decreased gradually with age. The duties of those in their $40 \mathrm{~s}$ were half that of workers in their $30 \mathrm{~s}$; for those in their $50 \mathrm{~s}$, they were half that of workers in their $40 \mathrm{~s}$.

For female pediatricians, more than half (58.4\%) reported they have refused overnight duties; $63.5 \%$ had at least one child at home [2]. In Japan, female public employees are offered paid maternity leave for 6 weeks before and after delivery, which obviously affects their time to work any overnight shifts. As a result, the average frequency of overnight work for female pediatricians was assumed to be a third of the male pediatrician rate.

Further, three categories relating to the number of night shifts worked each month were created conceptually for male pediatric hospitalists aged in the $20 \mathrm{~s}$ and $30 \mathrm{~s}$. They were 10 (classed as type10), 8 (type 8 ) and 6 (type 6), respectively. Type 10 means that in the hospital male pediatricians of $20 \mathrm{~s}$ and $30 \mathrm{~s}$ had a duty of 10-timenight-works in a month. Taking with former assumptions, for type 10, the monthly number of night-duty of males aged in their $40 \mathrm{~s}$ should be 5 , those in their $50 \mathrm{~s}$ is 2.5 ; in type 8, 4 for workers in their $40 \mathrm{~s}$ and 2 for $50 \mathrm{~s}$; in type 6, 3 for $40 \mathrm{~s}$ and 1.5 for $50 \mathrm{~s}$.

In addition, a typical hospital with 10 pediatricians is assumed to have a similar age distribution as that of Japan in general. Therefore, a typical hospital should have 5 pediatricians in their $20 \mathrm{~s}$ and $30 \mathrm{~s}$ ( 3 male, 2 female), 3 in their $40 \mathrm{~s}$ ( 2 male, 1 female), 1 aged in their $50 \mathrm{~s}$ (male), and 1 pediatrician aged 60 or greater. Finally, we ignored the difference in working hours for weekends and holidays, and considered a month to be 30 days.

Taking the above assumptions into account, for the type 10 classification, the ideal hospital with 10 pediatricians should have 50.8 [ $=3 \times 10$ (male $20 \mathrm{~s}$ and $30 \mathrm{~s}$ ) $+2 \times 10 \times 0.3$ (female $20 \mathrm{~s}$ and $30 \mathrm{~s}$ ) $+2 \times 5$ (male $40 \mathrm{~s}$ ) + $1 \times 5 \times 0.3$ (female $40 \mathrm{~s}$ ) $+1 \times 2.5$ (male $50 \mathrm{~s}$ )] overnight workers per month, 40.7 for type 8 , and 30.5 for type 6 , respectively. Because ideal hospitals had 10 pediatricians, this means that the average number of overnight shifts per month for a pediatrician is 5.08 [=50.8/10] for type 10, 4.07 for type 8 , and 3.05 for type 6 , respectively. Finally, the available number of pediatricians per night in hospitals with 10 pediatricians can be assumed to range from 1.0 [type $6=30.5 / 30$ ] to 1.7 [type $10=50.8 / 30$ ] (Table 1 ).

The above assumptions were made thinking of ideal working conditions. The following calculations would partially take actual conditions into consideration. Since the number of pediatric hospitalists was 9,308 and assuming that all hospitals with pediatricians that can work night shifts have 10 pediatricians, then the number of this ideal type of hospital equates to 931 [=9308/10]. Because the total population of Japan in 2011 was $127,799,000$, with a child population of $13.1 \%$ [6], during the night, one ideal hospital should cover a total population of 137,271 [=127,519,000/931], or 17,982 children.

According to a report from pediatric department of a hospital in the central part of Tokyo, 20.5 Japanese children per 1000 children visit an emergency department in a month [7]. By ignoring the temporal inequality in the distribution of patient visits, the number of visitors to an emergency department during the night per month 
Table 1. Comparison of number of night duties night per physician per month between ideal hospital (type 10, 8, 6) and real situation.

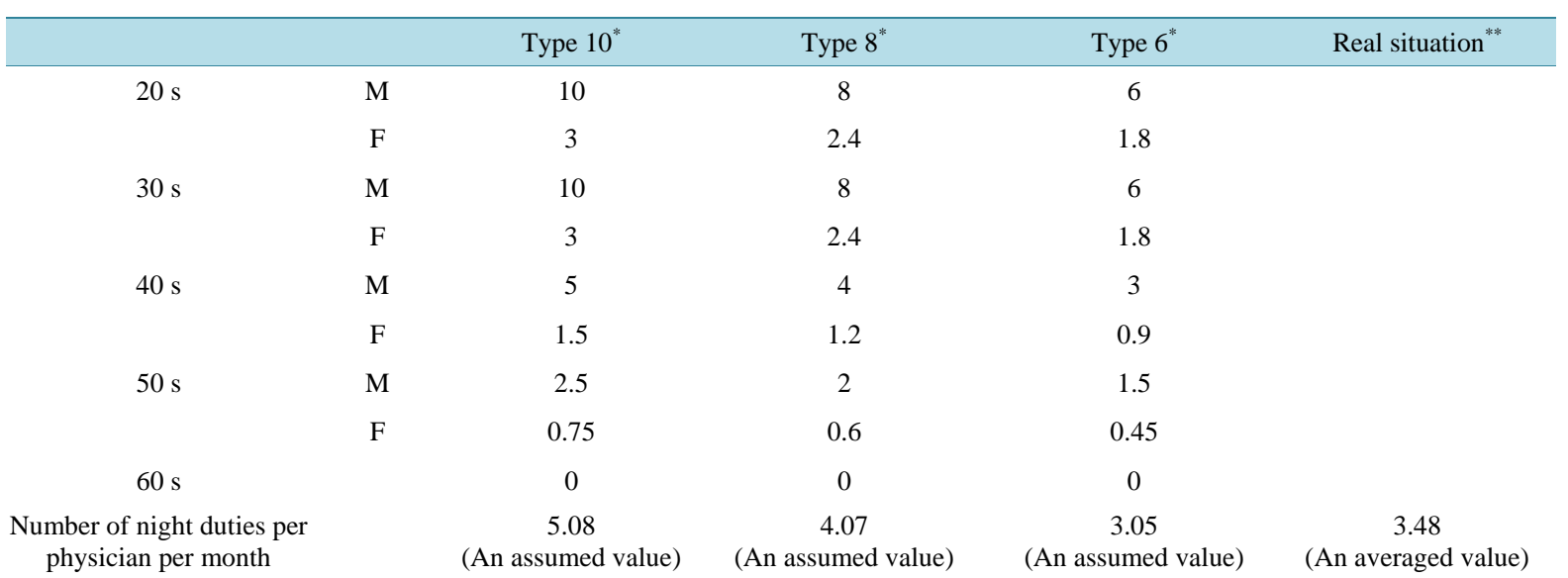

*Ideal hospital composed of 10 physicians of 3 males and 2 females of $20 \mathrm{~s}$ and $30 \mathrm{~s}, 2$ males and 1 female of $40 \mathrm{~s}, 1$ male of $50 \mathrm{~s}$, and 1 physician of $60 \mathrm{s.}{ }^{* *}$ an average of 2.6 physicians per hospital.

is estimated to be 10.3 [ $=20.5 / 2$ ] per 1000 children, and that each night the figure is 0.34 per 1000 children [=10.3/30]. Thus, the number of pediatric patients visiting an ideal hospital where 10 pediatricians are working is calculated to be 6.1 children [ $=0.34 \times 17982 / 1000]$. The rate of hospitalization from Japanese emergency departments has been reported by several hospitals with 10 or more pediatricians, as being $4.9 \%$ to $9.2 \%$ [7]-[10]. Therefore, under ideal conditions, 1 to 1.7 pediatricians would take care of 6.1 patients with 0.3 [=6.1 × 0.049]$0.6[=6.1 \times 0.092]$ new hospitalizations per night, in addition to caring for inpatients.

However, the actual number of hospitals providing pediatric services in 2011 was reported to be 2745 [11] (compared with 931 in the present assumption), and the average number of pediatricians in hospitals providing pediatric services in 2004 was 2.6 [12] (10 in the assumption). Further, in 2005 nearly 90\% of hospitals providing pediatric services had fewer than 10 pediatricians [13] ( $0 \%$ in the assumption). The ideal conditions assumed in the current study are substantially different from the actual situation in recent years. In 2008, the average number of overnight shifts worked by pediatricians per month has been reported as 3.48 [14] (as opposed to 5.08 for type10, 4.07 for type 8, and 3.05 for type6 in the present assumption) (Table 1).

According to the report published in 2013 [15], among 1111 hospitals professing to take care of sick children, $26.0 \%$ has no beds for hospitalization, and $65 \%$ complained the shortage of pediatricians.

\section{Discussion}

We initially would evaluate the validity of the type 6,8 and 10 classifications using specific assumptions. Type 6 was one weekday nighttime duty plus two weekend night duties per month; type 10 included night work every 3 days. Most pediatric hospitalists also had daytime duties, except those relating to the days after night work. According to the Japanese Nursing Association, the mean number of nights worked each month is 4.5 nights for nurses who work whole nights [16]. In relation to taxi drivers, according to the Act from the MHWL, the number of continuous 16 hour shifts is restricted to seven times per month [17]. For the type10 and type 8 classifications, this could translate to 8 to 10 continuous 24 hour periods spent at work per month. This frequency is unlikely to be acceptable, not only because of the physical and mental burden, but also from a medical safety perspective. As mentioned previously, the average number of overnight shifts worked by pediatricians each month is 3.48 [14]. Type 6 (the calculated average number of overnight shifts per one month; 3.05) may be consistent with actual night work burdens as well as ideal hospital conditions. However, interestingly, $65 \%$ of hospitals providing pediatric service complained the shortage of pediatricians. Burden especially for that during night among most current pediatric hospitalists is supposed to be hard. Some pediatric hospitalists might be working with type 8 or type10 situation.

The current calculation revealed that, under ideal conditions, 1 to 1.7 pediatricians would take care of 6.1 patients with 0.3 - 0.6 new hospitalizations per night, in addition to caring for inpatients. This nighttime working situation might be acceptable to many pediatricians. Thus, the recommendations made by JPS are logical and 
suitable including night burden. However, we found the calculated ideal conditions established using the study assumptions, i.e. all hospitals where pediatricians are working should have 10 pediatricians, is far from the actual current situation. It is obvious that demand supply balance on child medical care especially during night is disrupted. To compare with the assumed ideal conditions, current pediatric hospitalists are working in many hospitals with a few colleagues. Such a situation fails to provide the adequate services during the night for sick children. In 2005, JPS reported that the inadequate working situation of pediatric hospitalists would be improved by increasing the number of pediatric hospitalists from 6500 to 7560 [18], which have been accomplished, now. Something beyond expectations must be occurred. Concentration of pediatric hospitalists, which has been encouraged by JPS, might be insufficient. This should be put forward more and more, although barriers such as unequal population distribution, landform, the way of transportation, etc. exist. During the transient period until the completion of concentration of pediatric hospitalists, however, the decrease of demand on pediatricians should also be advanced. To solve this problem, two solutions could come to mind; the increase of physicians and the decrease of patients. An involvement of non-pediatric physicians in the medical care of children during the night could be the solution for the former problem, and the education for parents might be for the latter one. Many local governments in Japan have stated they will manage public hospitals so child patients can always be cared for by pediatricians. This statement reflects the JPS declaration about children receiving care from pediatricians [1]. In fact, the number of pediatricians per 100,000 child population less than 15 years old (80 per $100,000)$ is higher in Japan than the corresponding population in the USA (57 per 100,000) [19]. However, the current analysis showed that while this statement might be the ideal scenario, it is also unrealistic at the current situation of insufficient concentration of pediatric hospitalists. Support from non-pediatric physicians might partly contribute to the solution for the current imbalance between the availability of pediatricians and the social demands for them. Of course, improvements to the training system for non-pediatric physicians, so they can provide appropriate primary child care, should be encouraged.

In addition, public education will be indispensable for resolving the frustrations of both parents and pediatricians. Parents in Japan should know about the limitations related to the available capacity of pediatricians. They need to accept that their children can be cared for adequately by non-pediatric physicians, who have been trained to provide primary medical care to children. Public awareness programs are urgently needed to expose these realities, and to ease the current social problems relating to the apparent shortage of pediatricians. It should be noted that a movement to support pediatric hospitalists by mothers have been launched in 2007 [20].

\section{Conclusion}

To solve the current supply-demand mismatch on medical care for children during night, an involvement of non-pediatric physicians in the medical care of children during the night is indispensable. Also, parents in Japan need to know the limitations relating to the available capacity of pediatricians, and accept that their children can be cared for adequately by non-pediatric physicians, who have been trained to provide primary medical care to children.

\section{Conflicts of Interest}

The author has no conflict of interest on this manuscript.

\section{References}

[1] Japan Pediatric Society (2013) The Conception on Medical Services for Children in Japan. http://www.jpeds.or.jp/pdf/kyukyu.pdf

[2] Tanaka, T. (2003) Pediatric Emergency Medicine and Working Environment of Female Doctors (in Japanese). Nihon Shounika Gakkaizasshi, 107, 1478-1485.

[3] Kohyama, J. (2011) Neurochemical and Neuropharmacological Aspects of Circadian Disruptions: An Introduction to Asynchronization. Current Neuropharmacology, 9, 330-341. http://dx.doi.org/10.2174/157015911795596522

[4] Moline, M.L., Pollak, C.P., Monk, T.H., Lester, L.S., Wagner, D.R., Zendell, S.M., et al. (1992) Age-Related Differences in Recovery from Simulated Jet Lag. Sleep, 15, 28-40.

[5] Portal Site of Official Statistics of Japan (2013) Available from the No. 39 of the Following Web Address: http://www.e-stat.go.jp/SG1/estat/List.do?lid=000001084609

[6] Portal Site of Official Statistics of Japan (2013) http://www.stat.go.jp/data/jinsui/2011np/\#a05k23-a 
[7] Ishida, Y., Ohde, S., Takahashi, O., Deshpande, G.A., Shimbo, T., Hinohara, S., et al. (2012) Factors Affecting Health Care Utilization for Children in Japan. Pediatrics, 129, e113-119. http://dx.doi.org/10.1542/peds.2011-1321

[8] Tokushima Red Cross Hospital (2013) http://www.tokushima-med.jrc.or.jp/kakuka/a08_shoni.htm

[9] Ishikawa Prefectural Central Hospital (2013) http://www.pref.ishikawa.jp/ipch/syouninaikacurriculumsyousai.html

[10] SaiseikaiYokohamashiNanbu Hospital (2013) http://www.nanbu.saiseikai.or.jp/post/depts/pediatrics.html

[11] Ministry of Health, Labor and Welfare (2013) http://www.mhlw.go.jp/toukei/saikin/hw/iryosd/11/dl/1-1.pdf

[12] Ehara, A. (2013) The Change of Number of Pediatricians in a Hospital. http://pediatrics.news.coocan.jp/my_paper/hensen1.html

[13] Japan Pediatric Society (2013) Future Demands on Pediatric Hospitalists. http://www.mhlw.go.jp/shingi/2005/04/s0406-6a.html

[14] Ministry of Health, Labor and Welfare (2013) Report on the Relief of Burden of Hospitalists. http://www.mhlw.go.jp/shingi/2009/05/dl/s0520-4b.pdf

[15] Japan Pediatric Society (2013) The Current Status of Pediatrics in Hospitals. http://www.jpeds.or.jp/saisin/saisin_130219_1_1.pdf

[16] Japanese Nursing Association (2013) To Relief Night-Work Burden and Long-Time Labor. http://www.nurse.or.jp/nursing/practice/shuroanzen/jikan/pdf/yakinfutan.pdf

[17] Ministry of Health, Labor and Welfare (2013) The Summary on the Improvement of Working Conditions of Taxi Drivers. http://www.mhlw.go.jp/new-info/kobetu/roudou/gyousei/kantoku/dl/040330-12.pdf.

[18] Japan Pediatric Society (2013) Future Demands on Pediatric Hospitalists. http://www.jpeds.or.jp/pdf/syouni_future.pdf

[19] Ministry of Health, Labor and Welfare (2013) Report on the Effective Use of Medical Resources of Pediatricians and Obstetricians. http://www.mhlw.go.jp/shingi/2005/12/s1212-10g.html

[20] A Group to Protect Pediatric Hospitalists in Hyogo Prefectural Kaibara Hospital (2013) http://mamorusyounika.com/ 